\title{
Evidence-Based Robust Optimisation of Space Systems with Evidence Network Models
}

\author{
Gianluca Filippi \\ Department of Mechanical \& Aerospace Engineering \\ University of Strathclyde, Glasgow, UK \\ Email: g.filippi@ strath.ac.uk
}

\author{
Mariapia Marchi \\ ESTECO SpA, Trieste, IT \\ Email: marchi@esteco.com
}

\author{
Massimiliano Vasile \\ Department of Mechanical \& Aerospace Engineering \\ University of Strathclyde, Glasgow, UK \\ Email: massimiliano.vasile@strath.ac.uk \\ Paolo Vercesi \\ ESTECO SpA, Trieste, IT \\ Email: vercesi@esteco.com
}

\begin{abstract}
The paper presents an approach to optimise complex systems in space systems engineering, accounting for epistemic uncertainty. Uncertainty is modelled with Dempster-Shafer theory of Evidence and the space system as a network of connected components. A constrained min-max problem is then solved, with a memetic algorithm, to deliver a robust design point. Starting from this robust design point a sequence of evolutionary optimisation steps are used to reconstruct an approximation of the Belief and Plausibility curves associated to a particular design solution. The constrained min-max approach and the evolutionary reconstruction of the Belief and Plausibility curves are tested on a realistic case study of space systems engineering.
\end{abstract}

\section{INTRODUCTION}

Space systems engineering aims at designing, developing and verifying a system that can operate in space. This system is made of a number of interconnected components or subsystems, each of which performs a number of tasks, has inputs and outputs shared with other components and is qualified by a number of attributes. The failure or under-performance of one or more components affects the whole system and can lead to a system failure.

In the early design phase, one main point of concern is to evaluate the overall performance or attribute of the whole system. Typically a performance metric is the overall mass of the system but other attributes can become key performance indicators, like the data volume or the power output.

One critical aspect in the evaluation of particular systems engineering solution is the reliability of the value associated to a given performance metric. In fact, due to the uncertainty in the requirements, operational conditions, model parameters, component characteristics, etc. a deterministic design solution might not provide a reliable result.

The uncertainty introduced at an early design stage is mainly epistemic and require an appropriate treatment [1][2]. This paper starts from the concept of Evidence Network Models, recently introduced in [3], and extends previous work to introduce constraints in the system definition and operations. In Evidence Network Models, epistemic uncertainty are modelled as Belief functions and two values (Belief and Plausibility) are associated to the value of the system performance [4].

The resulting system optimisation under uncertainty process is composed of the solution of a constrained bi-level minmax optimisation problem followed by the reconstruction of the Belief and Plausibility on the value of the performance metric. The constrained min-max problem is a variation of the unconstrained approach presented in [5] and its solution is here addressed with a modification of Inflationary Differential Evolution [6] implemented in the software code MPAIDEA [7]. MPAIDEA is then used to provide an approximation to the Belief curves following the $\mathrm{h}$-decomposition process proposed in [8]. The paper is structured as follows. After introducing some fundamentals on Evidence Network Models, the paper presents the strategy to solve the constrained min-max problem and the h-decomposition approach. Results on a real-world test case will then follow.

\section{Evidence Network Models}

A generic complex system can be represented as a network, where each node is a subsystem and information is shared through links between subsystems. We can then define a function $F$ as

$$
F(\mathbf{d}, \mathbf{u})=\sum_{i=1}^{N} g_{i}\left(\mathbf{d}, \mathbf{u}_{i}, \mathbf{h}_{i}\left(\mathbf{d}, \mathbf{u}_{i}, \mathbf{u}_{i j}\right)\right)
$$

where $N$ is the number of subsystems involved, $\mathbf{h}_{i}\left(\mathbf{d}, \mathbf{u}_{i}, \mathbf{u}_{i j}\right)$ is the vector of scalar functions $h_{i j}\left(\mathbf{d}, \mathbf{u}_{i}, \mathbf{u}_{i j}\right)$ where $j \in$ $J_{i}$ and $J_{i}$ is the set of indexes of nodes connected to the $i$-th node; $\mathbf{u}_{i}$ are the uncertain variables of subsystem $i$ not shared with any other subsystem and $\mathbf{u}_{i j}$ are the uncertain variables shared among subsystems $i$ and $j$. Please note that according to our notation $\mathbf{u}_{i j}=\mathbf{u}_{j i}$ and the functions $g_{i}(\cdot, \cdot, \cdot)$ represent quantities computed by the governing equations of 


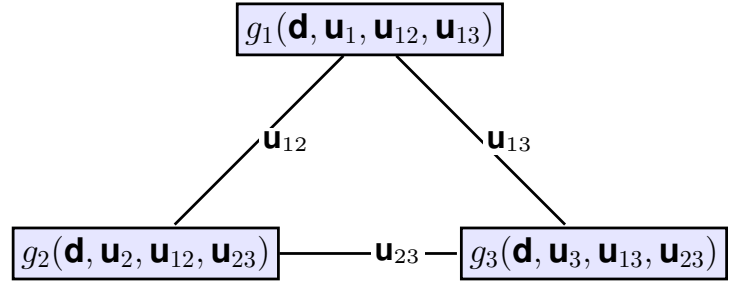

Fig. 1. Evidence Network Model of a generic system $F$ composed of three sub-systems with coupled variables $u_{12}, u_{13}$ and $u_{23}$.

the different subsystems. In a fully connected network as in Figure 1 the function $F$ is:

$$
\begin{aligned}
F(\mathbf{d}, \mathbf{u})= & g_{1}\left(\mathbf{d}, \mathbf{u}_{\mathbf{1}}, h_{12}\left(\mathbf{d}, \mathbf{u}_{\mathbf{1}}, \mathbf{u}_{\mathbf{1 2}}\right), h_{13}\left(\mathbf{d}, \mathbf{u}_{\mathbf{1}}, \mathbf{u}_{\mathbf{1 3}}\right)\right)+ \\
& g_{2}\left(\mathbf{d}, \mathbf{u}_{\mathbf{2}}, h_{21}\left(\mathbf{d}, \mathbf{u}_{\mathbf{2}}, \mathbf{u}_{\mathbf{1 2}}\right), h_{23}\left(\mathbf{d}, \mathbf{u}_{\mathbf{2}}, \mathbf{u}_{\mathbf{2 3}}\right)\right)+ \\
& g_{3}\left(\mathbf{d}, \mathbf{u}_{\mathbf{3}}, h_{31}\left(\mathbf{d}, \mathbf{u}_{\mathbf{3}}, \mathbf{u}_{\mathbf{1 3}}\right), h_{32}\left(\mathbf{d}, \mathbf{u}_{\mathbf{3}}, \mathbf{u}_{\mathbf{2 3}}\right)\right)
\end{aligned}
$$

We then call $\mathbf{u}_{i}$, uncoupled variables because they influence only one subsystem and $\mathbf{u}_{i j}$ coupled variables because they influence two subsystems. Hence, for the example in Figure 1 , the uncertain vector can be ordered as

$$
\mathbf{u}=\left[\mathbf{u}_{1}, \mathbf{u}_{2}, \mathbf{u}_{3}, \mathbf{u}_{12}, \mathbf{u}_{13}, \mathbf{u}_{23}\right]^{T} .
$$

In the following we will study only the case of functions $g_{i}(\cdot, \cdot, \cdot)$ that are always positive semi-definite and monotonic with respect to each function $h_{i k}$.

Given a design, or decision, value $\tilde{\mathbf{d}} \in \mathrm{D}$ we will call worst case scenario the vector $\underline{\mathbf{u}}$ that corresponds to the maximum of $F$ over the space $U$ :

$$
\underline{\mathbf{u}}=\underset{\mathbf{u} \in U}{\operatorname{argmax}} F(\widetilde{\mathbf{d}}, \mathbf{u}) .
$$

Likewise we can call best case scenario the quantity:

$$
\overline{\mathbf{u}}=\underset{\mathbf{u} \in U}{\operatorname{argmin}} F(\widetilde{\mathbf{d}}, \mathbf{u}) .
$$

We can now define an event in the space $U$, or a proposition on the value of $F$, as the set $A$ such that:

$$
A=\{\mathbf{u} \in U \mid F(\mathbf{d}, \mathbf{u}) \leq \nu\} .
$$

From this definition it is clear that for every design $\mathbf{d} \in D$ the worst case scenario corresponds to $A=U$, because $\nu=$ $\max _{\mathbf{u} \in U} F(\mathbf{d}, \mathbf{u})$, and analogously the best case scenario has zero measure. Each uncoupled uncertain vector $\mathbf{u}_{i}$ is defined over a set of boxes named $\Theta_{i}=\cup_{k} \theta_{k, i}$ and each coupled uncertain vector $\mathbf{u}_{i j}$ is defined over the set of boxes $\Theta_{i j}=$ $\cup_{k} \theta_{k, i j}$. We define the set

$$
\Theta=\bigcup_{i} \theta_{i}=\left(\times_{i=1}^{m_{u}} \Theta_{i}\right) \times\left(\times_{i, j=1}^{m_{c}} \Theta_{i j}\right)
$$

where $m_{u}$ is the number of uncoupled uncertain vectors (equal to the number of subsystems) and $m_{c}$ is the number of coupled uncertain vectors. In this context, the hyperpower set

$$
D^{\Theta}=(\Theta, \cup, \cap)
$$

is the set composed of the elements of $\Theta$, their union and intersection. In the following the space $U:=D^{\Theta}$. We can then define two quantities associated to the belief in the occurrence of the event $A$ :

$$
\begin{aligned}
& \operatorname{Bel}(A)=\sum_{\theta \subset A, \theta \in U} b p a(\theta) \\
& \operatorname{Pl}(A)=\sum_{\theta \cap A \neq 0, \theta \in U} b p a(\theta)
\end{aligned}
$$

where $\operatorname{bpa}(\theta)$ is the basic probability assignment associated to $\theta$, an element of the power set [4]. It is important to note that if the $h_{i j}$ functions were known with certainty the nodes composing the network would be decoupled and statistically independent. We also note that in order to identify if a $\theta$ is fully included in $A$ we need to find the maximum of $F$ with respect to $\mathbf{u} \in \theta$. Likewise an intersection with $A$ requires computing the minimum of $F$ with respect to $\mathbf{u} \in \theta$. Given that the subsets $\theta$, their unions and intersections come from a cross product, it is clear that the number of maximisation and minimisation increases exponentially with the number of dimensions. The computation of the Belief $\mathrm{Bel}$ (Plausibility $P l$ respectively) in the occurrence of $A$ is, therefore, an exponentially complex operation. In the following section a technique is proposed to compute an approximation to (7) by exploiting some of the properties of the ENM listed above. In particular we will exploit the following three properties:

1) The contribution of the coupled variable $\mathbf{u}_{i j}$ to the value $F$ manifests through the scalar functions $h_{i j}$ and $h_{j i}$.

2) All $g_{i}$ functions are positive semi-definite.

3) All $g_{i}$ functions are monotonically increasing with respect to $h_{i j}$ for every $j$.

\section{CONSTRAined MinmaX}

The approach to the design of complex systems under uncertainty proposed in this paper, requires the solution of one or more constrained min-max optimisation problems. The solution to this class of problem is here approached with a constrained variant of MPAIDEA, an adaptive version of Inflationary Differential Evolution [7]. This section describes only the strategy to handle constraints in the min-max version of MPAIDEA. More details on the approach to the solution of unconstrained min-max problems with Inflationary Differential Evolution can be found in [9].

\section{A. Memetic Approach}

The min-max algorithm proposed in this paper iteratively solves a bi-level optimisation, first minimising over the design vector $\mathbf{d}$ (outer loop) and then maximising over the uncertainty vector $\mathbf{u}$ (inner loop). The inner loop provides solutions that satisfy the constraint, while the outer loop maintains the constraint satisfaction while minimising the cost function $F$. The constraint handling procedure, summarised in Algorithm 1, implements the following steps:

- Initialisation of a population of $\mathbf{d}$ and $\mathbf{u}$ vectors; 
- While the number function evaluations is lower than $N_{\text {feval }}^{\max }$ function evaluations, do the following

- [Outer Loop] Constrained minimisation of the objective function over the design space, evaluating the cost function $F$ over all the uncertainty vectors stored in an archive $A=A_{u} \cup A_{c}$ :

$$
\begin{aligned}
& \min _{\mathbf{d} \in D \wedge \mathbf{u} \in A} F(\mathbf{d}, \mathbf{u}) \\
& \text { s.t. } \\
& \max _{\mathbf{u} \in A} C(\mathbf{d}, \mathbf{u}) \leq 0
\end{aligned}
$$

- [Inner Loop] Constrained maximisation of the cost function $F$ over the uncertain parameters $\mathbf{u}$ and parallel maximisation of the constraint violation over the uncertainty space:

$$
\begin{aligned}
& \max _{\mathbf{u} \in U} F\left(\mathbf{d}_{\text {min }}, \mathbf{u}\right) \\
& \text { s.t. } \\
& C(\mathbf{d}, \mathbf{u}) \leq 0 \\
& \quad \max _{\mathbf{u} \in U} C\left(\mathbf{d}_{\text {min }}, \mathbf{u}\right)
\end{aligned}
$$

$\mathbf{u}_{a, F}=\operatorname{argmax}_{u \in U} F\left(\mathbf{d}_{\text {min }}, \mathbf{u}\right)$ is added to the archive $A_{u}$ and $\mathbf{u}_{a, C}=\operatorname{argmax}_{u \in U} C\left(\mathbf{d}_{\min }, \mathbf{u}\right)$ is added to $A_{c}$ if $\max _{\mathbf{u} \in U} C(\mathbf{d}, \mathbf{u})>0$. This approach pushes the optimiser to find design configurations that are feasible for all values of the uncertain variables. If a feasible solution cannot be found, the constraints are relaxed (line 15), in the Inner Loop, by computing the new constraint $C^{*}=C+\epsilon$ with $\epsilon$ the minimum constraint violation over $U$.

In the multi-objective optimisation:

- Cross-check of the final solutions and choice of the best design;

- Final maximisation over U.

\section{Decomposition Algorithm}

In order to reduce the computational complexity of the calculation of $\operatorname{Bel}(A)$ we propose a decomposition technique that exploits the three properties defined in the previous section. The decomposition algorithm aims at decoupling the subsystems over the uncertain variables in order to optimise only over a small sub-set of the Focal Elements (FEs) (Algorithm $2)$; this procedure requires the following steps:

1) Solution of the optimal worst case scenario problems (9),(10) and (11).

2) Maximisation over the coupled variables and computation of $m_{c}$ partial $\mathrm{Bel}_{c}(A)$ curves.

3) Maximisation over the uncoupled variables.

4) Reconstruction of the approximation $\widetilde{\operatorname{Bel}}(A)$.

The result of the solution of problems (9),(10) and (11), as presented in section III, are the values $\tilde{\mathbf{d}}$ and $\underline{\mathbf{u}}$, thus, in the following, the decomposition starts once $\tilde{\mathbf{d}}$ and $\underline{\mathbf{u}}$ are available. However, it has to be noted that a Belief curve can be computed for any arbitrary $\mathbf{d}$.

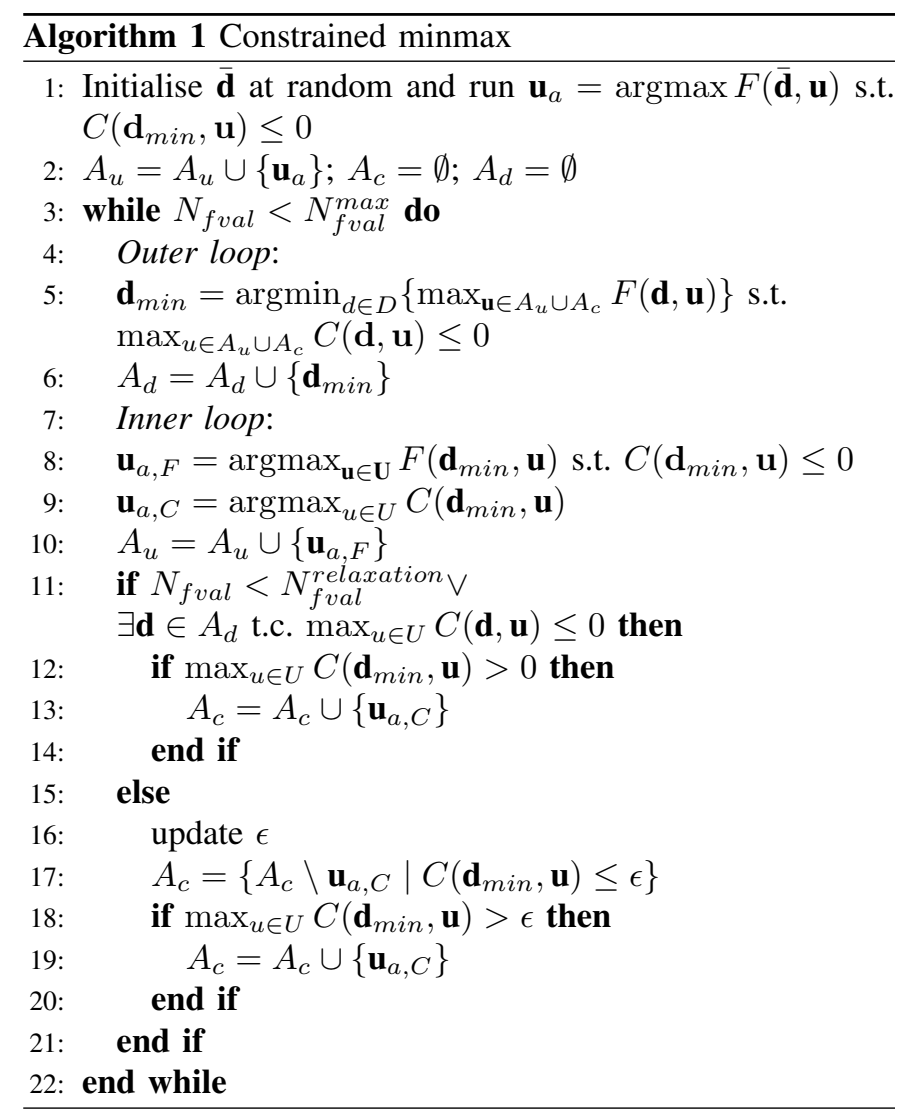

\section{A. Maximisation over the coupled variables and evaluation of the partial Belief curves}

For each coupled vector $\mathbf{u}_{i j}$ a maximisation is run over each FE $\theta_{k, i j} \subseteq \Theta_{i j} \subseteq U$, given $\mathbf{d}$ and keeping fixed all the other components of $\mathbf{u}$. Taking again the example in Figure 1 we have:

$\hat{\mathbf{u}}_{k, 12}=\underset{\mathbf{u}_{12} \in \theta_{k, 12}}{\operatorname{argmax}} F\left(\widetilde{\mathbf{d}}, \underline{\mathbf{u}}_{\mathbf{1}}, \underline{\mathbf{u}}_{\mathbf{2}}, \underline{\mathbf{u}}_{\mathbf{3}}, \mathbf{u}_{\mathbf{1 2}}, \underline{\mathbf{u}}_{\mathbf{1 3}}, \underline{\mathbf{u}}_{\mathbf{2 3}}\right), \forall \theta_{k, 12} \subset \Theta_{12}$
$\hat{\mathbf{u}}_{k, 13}=\underset{\mathbf{u}_{13} \in \theta_{k, 13}}{\operatorname{argmax}} F\left(\widetilde{\mathbf{d}}, \underline{\mathbf{u}}_{\mathbf{1}}, \underline{\mathbf{u}}_{\mathbf{2}}, \underline{\mathbf{u}}_{\mathbf{3}}, \underline{\mathbf{u}}_{\mathbf{1 2}}, \mathbf{u}_{\mathbf{1 3}}, \underline{\mathbf{u}}_{\mathbf{2 3}}\right), \forall \theta_{k, 13} \subset \Theta_{13}$
$\hat{\mathbf{u}}_{k, 23}=\underset{\mathbf{u}_{23} \in \theta_{k, 23}}{\operatorname{argmax}} F\left(\widetilde{\mathbf{d}}, \underline{\mathbf{u}}_{\mathbf{1}}, \underline{\mathbf{u}}_{\mathbf{2}}, \underline{\mathbf{u}}_{\mathbf{3}}, \underline{\mathbf{u}}_{\mathbf{1 2}}, \underline{\mathbf{u}}_{\mathbf{1 3}}, \mathbf{u}_{\mathbf{2 3}}\right), \forall \theta_{k, 23} \subset \Theta_{23}$

For the sake of simplicity we will indicate with

$$
F\left(\mathbf{u}_{i j}\right):=F\left(\tilde{\mathbf{d}}, \underline{\mathbf{u}}_{1}, \ldots, \mathbf{u}_{i j}, \ldots, \underline{\mathbf{u}}_{i+1 j}, \ldots\right) .
$$

We can then compute the partial belief associated only to the coupled variables with index $i j$ :

$$
\operatorname{Bel}\left(F\left(\mathbf{u}_{i j}\right)<\nu\right)=\sum_{\theta_{k, i j} \mid \max _{\mathbf{u}_{i j} \in \theta_{k, i j}} F\left(\mathbf{u}_{i j}\right) \leq \nu} b p a\left(\theta_{k, i j}\right) .
$$

The calculation of the partial belief can be found in Algorithm 2 , line 6 . Once the partial belief curve, for each coupled vector, is available, one can sample these curves, by taking a succession of $\nu=\left\{\nu_{1}, \ldots, \nu_{q}, \ldots, \nu_{N_{S}}\right\}$ values, and find 
the corresponding values of the coupled vectors $\widehat{\mathbf{u}}_{k, i j}^{q}$. These values will be used in the next step to decouple the functions $g_{i}$ and compute the maxima of each $g_{i}$ with respect to the uncoupled variables $\mathbf{u}_{i}$.

\section{B. Optimisation over the uncoupled vectors}

For each $\nu_{q}$, given a fixed value of the coupling functions, one can study each $g_{i}$ independently of the others. The idea is to run an optimisation for each function $g_{i}$ over only the uncoupled vector $\mathbf{u}_{i}$. With the example in Figure 1 in mind, having

$$
\hat{h}_{i j}^{q}\left(\mathbf{u}_{i}\right):=h_{i j}\left(\tilde{\mathbf{d}}, \mathbf{u}_{i}, \hat{\mathbf{u}}_{i j}^{q}\right)
$$

where $\hat{\mathbf{u}}_{i j}^{q}:=\hat{\mathbf{u}}_{k^{*}, i j}^{q}: k^{*}=\operatorname{argmax}_{k} F\left(\hat{\mathbf{u}}_{k, i j}^{q}\right)$, is one of the maxima of the maxima attained by the coupled variable $\mathbf{u}_{i j}$. For every FE $\theta_{k, i} \in \Theta_{i}$ we have:

$$
\begin{aligned}
& \hat{\mathbf{u}}_{k, 1}^{q}=\underset{\mathbf{u}_{1} \in \theta_{k, 1}}{\operatorname{argmax}} g_{1}\left(\widetilde{\mathbf{d}}, \mathbf{u}_{1}, \widehat{h}_{12}^{q}\left(\mathbf{u}_{1}\right), \widehat{h}_{13}^{q}\left(\mathbf{u}_{1}\right)\right), \forall \theta_{k, 1} \subset \Theta_{1} \\
& \hat{\mathbf{u}}_{k, 2}^{q}=\underset{\mathbf{u}_{2} \in \theta_{k, 2}}{\operatorname{argmax}} g_{2}\left(\widetilde{\mathbf{d}}, \mathbf{u}_{2}, \widehat{h}_{21}^{q}\left(\mathbf{u}_{2}\right), \widehat{h}_{23}^{q}\left(\mathbf{u}_{2}\right)\right), \forall \theta_{k, 2} \subset \Theta_{2} \\
& \hat{\mathbf{u}}_{k, 3}^{q}=\underset{\mathbf{u}_{3} \in \theta_{k, 3}}{\operatorname{argmax}} g_{3}\left(\widetilde{\mathbf{d}}, \mathbf{u}_{3}, \widehat{h}_{31}^{q}\left(\mathbf{u}_{3}\right), \widehat{h}_{32}^{q}\left(\mathbf{u}_{3}\right)\right), \forall \theta_{k, 3} \subset \Theta_{3}
\end{aligned}
$$

with the corresponding values $\hat{g}_{k, 1}^{q}, \hat{g}_{k, 2}^{q}$ and $\hat{g}_{k, 3}^{q}$.

\section{Complexity Analysis}

From the definition of the hyperpower set in (6) it is clear that the number of focal elements increases exponentially with the number of dimensions. Even if one limits the $U$ space to the sole $\Theta$ the total number of FEs for a problem with $m$ uncertain variables, each defined over $N_{k}$ intervals, is:

$$
N_{F E}=\prod_{k=1}^{m} N_{k}
$$

In terms of coupled and uncoupled uncertain vectors we can write:

$$
N_{F E}=\left(\prod_{i=1}^{m_{u}} \prod_{k=1}^{p_{i}^{u}} N_{i, k}^{u}\right)\left(\prod_{i=1}^{m_{c}} \prod_{k=1}^{p_{i}^{c}} N_{i, k}^{c}\right)
$$

where $p_{i}^{u}$ and $p_{i}^{c}$ are the number of components of the $i-t h$ uncoupled and coupled vector, respectively, and $N_{i, k}^{u}$ and $N_{i, k}^{c}$ are the numbers of intervals of the $k-t h$ components of the $i-t h$ uncoupled and coupled vector respectively. The total number of focal elements that needs to be explored in the decomposition is instead:

$$
N_{F E}^{D e c}=N_{s} \sum_{i=1}^{m_{u}} N_{F E, i}^{u}+\sum_{i=1}^{m_{c}} N_{F E, i}^{c}
$$

considering the vector of uncertainties ordered as

$$
\mathbf{u}=[\underbrace{\mathbf{u}_{1}, \ldots, \mathbf{u}_{m_{u}}}_{\text {uncoupled }}, \underbrace{\mathbf{u}_{1}, \ldots, \mathbf{u}_{m_{c}}}_{\text {coupled }}]
$$

where and $N_{s}$ is the number of samples in the partial belief curves, $N_{F E, i}^{c}=\prod_{k=1}^{p_{i}^{c}} N_{i, k}^{c}$ and $N_{F E, i}^{u}=\prod_{k=1}^{p_{i}^{u}} N_{i, k}^{u}$. This means that the computational complexity to calculate the maxima of the function $F$ within the focal elements is polynomial with the number of subsystems and remains exponential for each individual uncoupled or coupled vector.

\section{Reconstruction}

Once all the maxima over the focal elements of the uncoupled variables are available for each sample $q$ one can calculate an approximation of $\operatorname{Bel}(F(\mathbf{d}, \mathbf{u})<\nu)$ as follows. From Eq. (14), for each sample $q$ the maximum associated to focal element $\theta_{k}=\theta_{k_{1}, 1} \times \theta_{k_{2}, 2} \times \theta_{k_{3}, 3}$, for $k=1, \ldots N_{F E, 1} \cdot N_{F E, 2} \cdot N_{F E, 3}$, using a positive semi-definite $g_{i}$, is:

$\max _{\left(\mathbf{u}_{1}, \mathbf{u}_{2}, \mathbf{u}_{3}\right) \in \theta_{k}} F\left(\tilde{\mathbf{d}}, \mathbf{u}_{1}, \mathbf{u}_{2}, \mathbf{u}_{3}, \hat{\mathbf{u}}_{12}^{q}, \hat{\mathbf{u}}_{13}^{q}, \hat{\mathbf{u}}_{23}^{q}\right)=\hat{g}_{k_{1}, 1}^{q}+\hat{g}_{k_{2}, 2}^{q}+\hat{g}_{k_{3}, 3}^{q}$

with associated basic probability assignment:

$$
b p a^{q}\left(\theta_{k}\right)=b p a\left(\theta_{k_{1}, 1}\right) b p a\left(\theta_{k_{2}, 2}\right) b p a\left(\theta_{k_{3}, 3}\right) \Delta B e l^{q}
$$

where $\Delta B e l^{q}=\prod_{i j} \Delta B e l_{i j}^{q}$ are the contributions of the partial belief curves in (13). In other words, the bpa of each $\theta_{k}$ is the product of all the bpa's of the FEs of each uncoupled variable scaled with the product of the belief values of the samples drawn from the partial belief curves (Line 18). The approximation of the belief is then computed as:

$$
\widetilde{\operatorname{Bel}}(F(\mathbf{d}, \mathbf{u}) \leq \nu)=\sum_{q} \sum_{k} b p a^{q}\left(\theta_{k}\right)
$$

If the decomposition drastically reduces the number of maximisations, the reconstruction still requires an exponential number of multiplications of bpa's. Thus, the computational cost of the reconstruction step would increase exponentially with the number of sub-systems if the full curve was required. In this case the number of times that (19) has to be evaluated is:

$$
N_{\text {evals }}=N_{s} \prod_{i=1}^{m_{u}} N_{F E, i}^{u} .
$$

If the decomposition is used to evaluate $\operatorname{Bel}(F(\mathbf{d}, \mathbf{u})<\nu)$, for a given $\mathbf{d}$ and a single threshold $\nu$, then a partial belief curve could be reconstructed only in a neighbourhood of $\nu$ at a reduced computational cost.

For a given sample $q$, consider the vector

$$
\hat{\mathbf{g}}_{i}^{q}=\left[\hat{g}_{i, 1}^{q}, \ldots, \hat{g}_{i, N_{F E, i}^{u}}^{q}\right]^{T}
$$

of all the maxima of a function $g_{i}$ over all the focal elements $\theta_{k, i}$ and the collection of vectors

$$
\begin{aligned}
\Gamma=\left[\gamma_{q i k}\right] & =1, \ldots, N_{S} \quad, \quad \gamma_{q i k}=\hat{g}_{k, i}^{q} \\
i & =1, \ldots, m_{u} \\
k & =1, \ldots, N_{F E, i}^{u}
\end{aligned}
$$


organised as in Table I. The approximated belief curve in Eq. (20) can be computed by taking the sum of the bpa's for every row of $\Gamma$ and then adding up all the rows.

Now, given $\nu$, one can filter out all the components $\hat{g}_{k, i}^{q}$ of each vector $\hat{\mathbf{g}}_{i}^{q}$ that satisfies the relationship:

$$
\hat{g}_{k, i}^{q}+\sum_{i=1}^{N_{U}} \min _{k} \hat{\mathbf{g}}_{i}^{q}>\nu .
$$

If condition (22) is applied to every vector in $\Gamma$ we obtain a new collection $\Gamma_{L}$. Symmetrically we can also construct the collection $\Gamma_{R}$ by filtering the vectors in $\Gamma$ with the following condition:

$$
\hat{g}_{k, i}^{q}+\sum_{i=1}^{N_{U}} \max _{k} \hat{\mathrm{g}}_{i}^{q}<\nu .
$$

The computation of $\operatorname{Bel}(F(\mathbf{d}, \mathbf{u}) \leq \nu)$ is realised by taking from each row of the two collections $\Gamma_{L}$ and $\Gamma_{R}$ the rows with the least amount of focal elements, i.e. the $\hat{g}_{i}^{\mathbf{q}}$ vectors with the lowest number of components, and form the new collection $\Gamma_{\nu}$. We can now calculate the approximated belief as in Eq.(20) but using the rows and columns of matrix $\Gamma_{\nu}$.

\section{TABLE I}

INFORMATION USED IN THE RECONSTRUCTION STEP

\begin{tabular}{l|llllll} 
& $s u b_{1}$ & $s u b_{2}$ & $\ldots$ & $s u b_{i}$ & $\ldots$ & $s u b_{m_{u}}$ \\
\hline sample $_{1}$ & $\hat{\mathbf{g}}_{1}^{1}$ & $\hat{\mathbf{g}}_{2}^{1}$ & $\ldots$ & $\hat{\mathbf{g}}_{i}^{1}$ & $\ldots$ & $\hat{\mathbf{g}}_{m_{u}}^{1}$ \\
$\ldots$ & $\ldots$ & $\ldots$ & $\ldots$ & $\ldots$ & $\ldots$ & $\ldots$ \\
$\operatorname{sample}_{q}$ & $\hat{\mathrm{g}}_{1}^{q}$ & $\hat{\mathrm{g}}_{2}^{q}$ & $\ldots$ & $\hat{\mathbf{g}}_{i}^{q}$ & $\ldots$ & $\hat{\mathbf{g}}_{m_{u}}^{q}$ \\
$\ldots$ & $\ldots$ & $\ldots$ & $\ldots$ & $\ldots$ & $\ldots$ & $\ldots$ \\
$\operatorname{sample}_{N_{s}}$ & $\hat{\mathrm{g}}_{1}^{N_{s}}$ & $\hat{\mathrm{g}}_{2}^{N_{s}}$ & $\ldots$ & $\hat{\mathrm{g}}_{i}^{N_{s}}$ & $\ldots$ & $\hat{\mathbf{g}}_{m_{u}}^{N_{s}}$ \\
\hline
\end{tabular}

The reconstruction computational cost after filtering is:

$$
N_{\text {evals }}=\sum_{q=1}^{N_{s}} \prod_{i=1}^{m_{u}} \operatorname{dim}\left(\hat{\mathbf{g}}_{\mathrm{i}}^{\mathrm{q}}\right), \quad \hat{\mathrm{g}}_{\mathrm{i}}^{\mathrm{q}} \in \Gamma_{\nu}
$$

\section{E. Simple example of the decomposition approach}

Consider the equation $F=g_{1}+g_{2}$ where $g_{1}=10 u_{1}^{2}+$ $\left|u_{2}\right| u_{5}^{2}+\frac{u_{6}^{4}}{100}+d_{1}\left|d_{2}\right|$ and $g_{2}=\left|u_{3}\right|+u_{4}^{2} \frac{\left|u_{5}\right|}{10}+u_{6}^{2}+\left|d_{1}\right|$ where there are two uncoupled vectors $\left(\mathbf{u}_{1}=\left(u_{1}, u_{2}\right)\right.$ and $\mathbf{u}_{2}=$ $\left.\left(u_{3}, u_{4}\right)\right)$ and one coupled vector $\left(\mathbf{u}_{12}=\left(u_{5}, u_{6}\right)\right)$. The minmax solution is $\widetilde{\mathbf{d}}=[-5,-5] \wedge \underline{\mathbf{u}}=[-5,-5,-5,-5,-5,-5]$ and the decomposition belief curves are presented in Fig. 2. Other simple test cases can be found in [3].

\section{CAse Study}

\section{A. Optimal Battery Sizing}

The minmax and h-decomposition approaches are here applied to the sizing of a battery on board a spacecraft during an orbital transfer from Geo Transfer Orbit to geostationary orbit (GEO)[11]. The goal is the constrained minimisation of the mass of the battery under epistemic uncertainty. The mass of the battery is dependent on the following design, uncertain and fixed parameters:

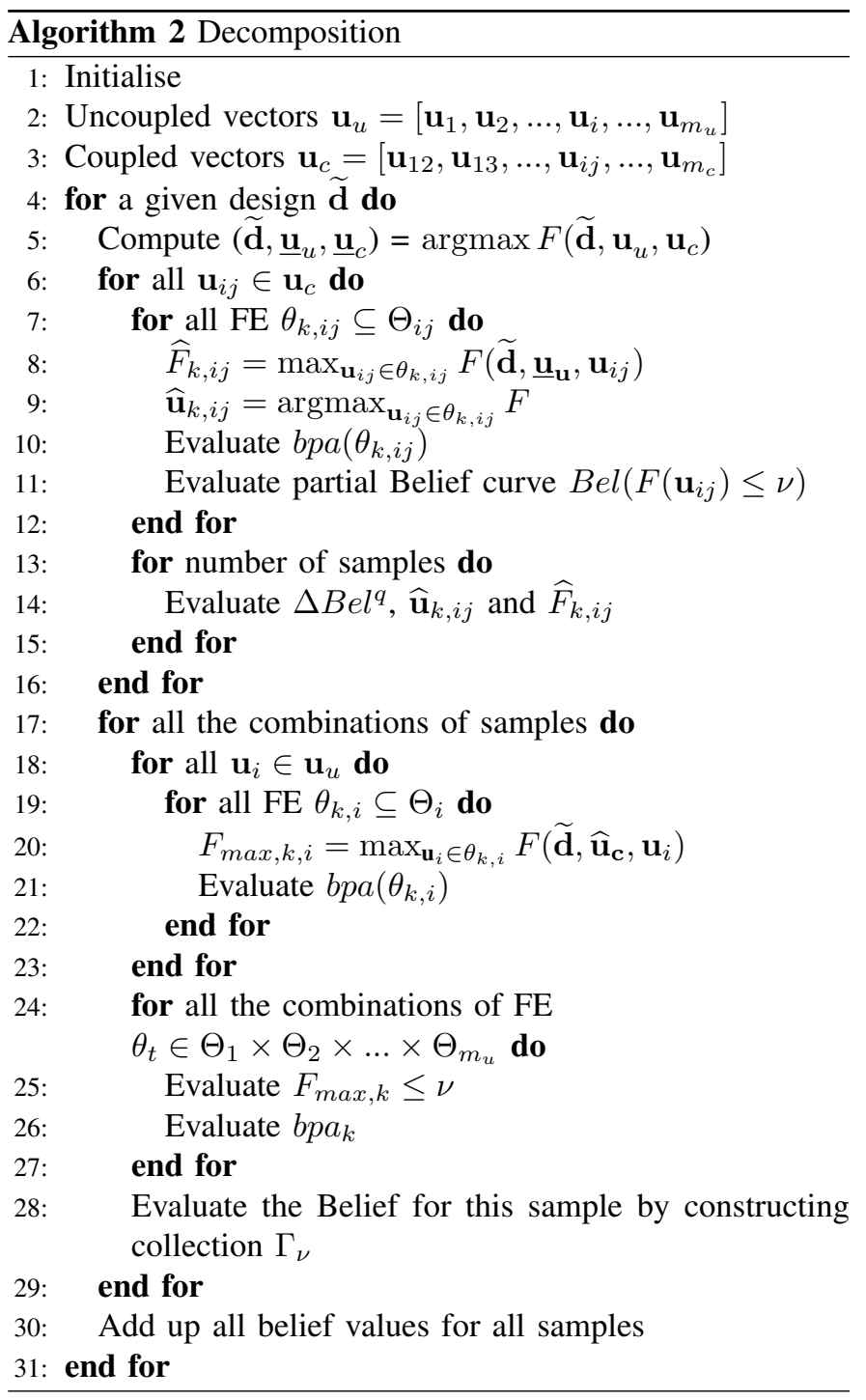

TABLE II

DESIGN PARAMETERS FOR BATTERY PROBLEM

\begin{tabular}{c|c|c}
\hline variable & symbol & value \\
\hline insertion time & $\mathrm{t}$ & {$[6939.87304 .8]$} \\
battery type & $\gamma$ & {$[0,1]$} \\
Bus voltage & $\Delta V_{B U S}$ & {$[0,5]$} \\
\hline
\end{tabular}

- 3 design parameters. Time of orbit insertion $t$, type of battery $\gamma$ and bus voltage $\Delta V_{\text {bus }}$ :

$$
\mathbf{d}=\left[t, \gamma, \Delta V_{b u s}\right]^{T} .
$$

Table II shows the design parameters with their associated range of variability: $t$ is given in Modified Julian Date (MJD) - for more details see [11] - and can be any day of 2019 at 7 a.m.; $\gamma$ ranges in four possible intervals - [0, $0.25),[0.25,0.5),[0.50 .75)$ and $[0.75,1]$ - corresponding to 4 battery types (see Table III).

- 31 uncertain parameters. Orbital parameters of five orbits $\boldsymbol{\alpha}$ - semimajor axis, a, eccentricity, e, Inclination, $i$, right ascension of the ascending node, $\Omega$, Argument 


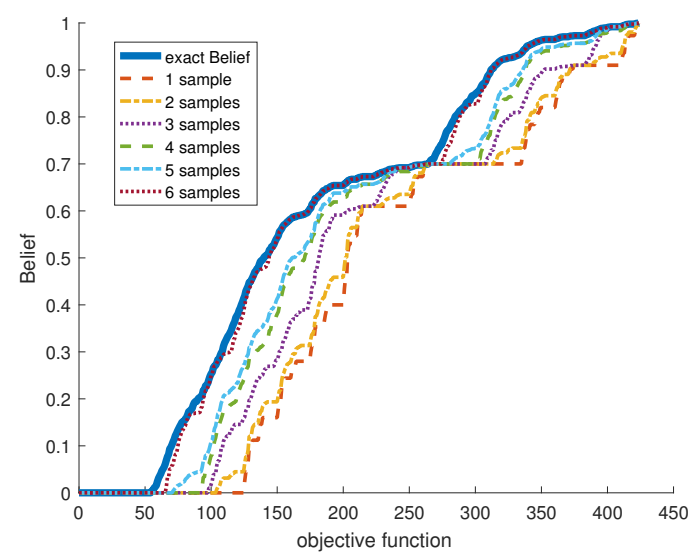

Fig. 2. Simple example of application of the decomposition approach

TABLE III

LOOKUP TABLE OF BATTERIES

\begin{tabular}{l|llll}
\hline BATTERY & A & B & C & D \\
\hline Cell capacity $C_{\text {cell }}(\mathrm{Ah})$ & 4.5 & 1.7 & 1.5 & 3.7 \\
Cell voltage $\Delta V_{\text {cell }}(\mathrm{V})$ & 4.1 & 4.2 & 4.2 & 4.1 \\
Cell Mass $m(\gamma)(\mathrm{kg})$ & 0.63 & 0.2 & 0.21 & 0.23 \\
Max DoD $D o D_{\max }(\%)$ & 80 & 75 & 75 & 75 \\
\hline
\end{tabular}

of Perigee, $\omega$ and True Anomaly, $\theta$ (for more details see [11])- and efficiency of the battery $\eta$ :

$$
\mathbf{u}=[\boldsymbol{\alpha}, \eta]=[\mathbf{a}, \mathbf{e}, \mathbf{i}, \boldsymbol{\Omega}, \boldsymbol{\omega}, \boldsymbol{\theta}, \eta]
$$

with:

$\mathbf{a}=\left[a_{1}, a_{2}, a_{3}, a_{4}, a_{5}\right]^{T}, \mathbf{e}=\left[e_{1}, e_{2}, e_{3}, e_{4}, e_{5}\right]^{T}, \mathbf{i}=$ $\left[i_{1}, i_{2}, i_{3}, i_{4}, i_{5}\right]^{T}, \boldsymbol{\Omega}=\left[\Omega_{1}, \Omega_{2}, \Omega_{3}, \Omega_{4}, \Omega_{5}\right]^{T}, \boldsymbol{\omega}=$ $\left[\omega_{1}, \omega_{2}, \omega_{3}, \omega_{4}, \omega_{5}\right]^{T}$, and $\boldsymbol{\theta}=\left[\theta_{1}, \theta_{2}, \theta_{3}, \theta_{4}, \theta_{5}\right]^{T}$.

For each uncertain variable, two possible intervals are given, both with $50 \%$ of probability. They are symmetrically arranged on either side of the nominal values in Table V; the interval dimensions are given by Table IV.

TABLE IV

UNCERTAIN INTERVALS

\begin{tabular}{lllllll}
\hline & $\mathrm{a}(\mathrm{km})$ & $\mathrm{e}(-)$ & $\mathrm{i}\left(^{\circ}\right)$ & $\Omega\left(^{\circ}\right)$ & $\omega\left(^{\circ}\right)$ & $\theta\left(^{\circ}\right)$ \\
\hline$\Delta u$ & $\pm 20 \mathrm{~km}$ & \pm 0.0012 & $\pm 0.07^{\circ}$ & $\pm 30^{\circ}$ & $\pm 0.5^{\circ}$ & $\pm 0.025^{\circ}$
\end{tabular}

- 10 fixed parameters. Engine ignition time and Liquid Apogee Engine (LAE) burn time per orbit (Table VI).

TABLE V

NOMINAL VALUE OF THE EPISTEMIC PARAMETERS FOR SSTL PROBLEM

\begin{tabular}{lllllll}
\hline orbit & $\mathrm{a}(\mathrm{km})$ & $\mathrm{e}(-)$ & $\mathrm{i}\left(^{\circ}\right)$ & $\Omega\left(^{\circ}\right)$ & $\omega\left(^{\circ}\right)$ & $\theta\left(^{\circ}\right)$ \\
\hline 1 & 68500.3 & 0.90 & 22.81 & 86.63 & 180.10 & 0.00 \\
2 & 73250.2 & 0.77 & 9.12 & 86.79 & 180.6 & 180.08 \\
3 & 86065.5 & 0.51 & 1.09 & 85.96 & 180.81 & 180.84 \\
4 & 49646.4 & 0.15 & 0.36 & 86.85 & 180.97 & 4.25 \\
5 & 42049.0 & 0.001 & 0.05 & 270 & 0.00 & 359.95 \\
\hline
\end{tabular}

TABLE VI

FIXED PARAMETERS

\begin{tabular}{lll}
\hline orbit & Time of Arrival [hrs.] & Burn duration [hrs.] \\
\hline 1 & 0.00 & 0.0 \\
2 & 24.7 & 0.6 \\
3 & 79.9 & 0.8 \\
4 & 114.7 & 0.6 \\
5 & 145.2 & 0.3 \\
\hline
\end{tabular}

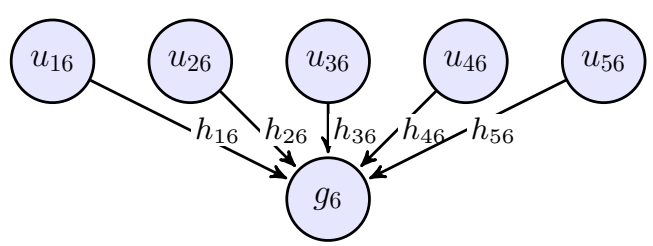

Fig. 3. Evidence Network Model of the optimal battery sizing problem.

The minmax problem is the constrained minimisation of the mass of the battery $\mathrm{M}$ under uncertainty:

$$
\min _{\mathbf{d} \in D} \max _{\mathbf{u} \in U} M(\mathbf{d}, \mathbf{u}) \quad \text { s.t. } \quad C(\mathbf{d}, \mathbf{u})<0 .
$$

M depends on the mass of the cell (Table III) and on the total number of cells of the battery:

$$
M=m(\gamma) N_{t o t}
$$

and $N_{t o t}$ is the product of the number of cells in series $N_{s}$ and in parallel $N_{p}$ :

$$
N_{\text {tot }}=N_{s} N_{p}=\frac{\Delta V_{B U S}}{\Delta V_{\text {cell }}} \frac{E}{D o D_{\max } \Delta V_{B U S} C_{\text {cell }}}+1
$$

where $E$, the Energy requirement, depends on the length of the eclipse periods evaluated from the input data, $\Delta V_{B U S}$ is the Bus voltage, $\Delta V_{\text {cell }}$ the cell voltage, $D o D_{\max }$ the maximum allowed Depth of Discharge, $C_{\text {cell }}$ the Cell Capacity [10]. The constraint is given by the maximum allowed Depth of discharge for each type of battery (Table III):

$$
C(\mathbf{d}, \mathbf{u}) \begin{cases}>0 & \text { if } D o D(\mathbf{d}, \mathbf{u})>D o D_{\max } \\ <0 & \text { if } \operatorname{DoD}(\mathbf{d}, \mathbf{u})<D o D_{\max }\end{cases}
$$

\section{B. Evidence Network Model of the Battery Sizing Problem}

The above-described battery sizing problem is modelled with an ENM assuming that each of the five orbits is a node of the network and all five nodes are connected to a $6^{\text {th }}$ node, the battery. The scheme of Fig. 3 illustrates this simple topology:

$$
M(\mathbf{d}, \mathbf{u})=\sum_{i=1}^{6} g_{i}\left(\mathbf{d}, \mathbf{u}_{i}, \mathbf{h}_{i}\left(\mathbf{d}, \mathbf{u}_{i}, \mathbf{u}_{i j}\right)\right)
$$

- 5 nodes evaluate the energy required by the spacecraft in the given orbits: $E_{i}\left(\mathbf{d}, a_{i}, e_{i}, i_{i}, \Omega_{i}, \omega_{i}, \theta_{i}\right)$ and have $g_{i}=0$ for $i=1,2, \ldots, 5$

- The 6th node sizes the mass of the battery: $g_{6}\left(\mathbf{d}, \eta, h_{16}, h_{26}, h_{36}, h_{46}, h_{56}\right)$ where $h_{i 6}=E_{i}$. 
The epistemic vector has been organised as:

$$
\mathbf{u}=[\underbrace{\mathbf{u}_{6}}_{\text {uncoupled }}, \underbrace{\mathbf{u}_{16}, \mathbf{u}_{26}, \mathbf{u}_{36}, \mathbf{u}_{46}, \mathbf{u}_{56}}_{\text {coupled }}]
$$

where: $\mathbf{u}_{6}=\eta$ and $\mathbf{u}_{i 6}=\left[a_{i}, e_{i}, i_{i}, \Omega_{i}, \omega_{i}, \theta_{i}\right]^{T}$ with $\mathrm{i}=$ $1, \ldots, 5$.

$\Theta_{6}=\cup_{k} \theta_{k, 6} \ni \mathbf{u}_{6}$ is the set of all the FE of the uncoupled vector $\mathbf{u}_{6}$ and $\Theta_{i 6}=\cup_{k} \theta_{k, i 6} \ni \mathbf{u}_{i 6}$ is the set of all the FE of the coupled vector $\mathbf{u}_{i 6}$ for $\mathrm{i}=1,2, \ldots, 5$.

In the ENM model of this problem the five orbits independently contribute to the mass as the uncertainty on the energy requirement manifest only through node 6 . Furthermore, node 6 is monotone with respect to the energy requirement of each orbit, independently of the uncertainty in the other orbits. Finally, the mass of the battery $M$ is proportional to the maximum energy requirement that depends on the maximum period of battery discharge. Because of the monotonic dependency of the discharge period on the uncertainty in each orbit, the maximum energy demand can be calculated directly from the minmax solution. Thus:

$M \propto E^{\max } \Rightarrow M \propto \max \left(E_{1}^{\max }, E_{2}^{\max }, E_{3}^{\max }, E_{4}^{\max }, E_{5}^{\max }\right)$.

With the specific orbital parameters used in this case study, the minmax algorithm always converges to a solution where the maximum energy requirement derives from the second orbit; thus only the second node of the presented ENM influences the sixth, the battery, through the exchange function $h_{26}$ : $g_{6}\left(\mathbf{d}, \eta, h_{26}\right)$ where $h_{26}=E_{2}$.

\section{Results}

The software MPAIDEA [7] has been used to provide the solution of both the min-max and the h-decomposition problems. The inner loop (maximisation over $\mathbf{u}$ ), the outer loop (minimisation over d) - explained in Section III - and also each optimisation for the decomposition approach have been set with a single population and a maximum number of function evaluations $N_{F}^{\max }=1000$ while the total number of function evaluations for whole the min-max loop is $N_{F}^{\max , t o t}=10^{5}$; the problems have been run multiple times obtaining the same results.

1) Min-Max: The minmax solution for the optimal battery sizing is $\widetilde{d}=[59, D, 36.9]^{T}$ with a corresponding battery mass of $126.3 \mathrm{~kg}$. For $t=59$ the mission is most affected by uncertainty, as shown in Figure 4a and Figure 4b. The Figures show the influence of the uncertainty for all the possible missions in the year 2019. The blue curves correspond to the nominal orbits in Table $\mathrm{V}$, while the red ones represent the maximisation over the uncertain parameters of the energy requirement (Figure 4a) and time of eclipse (Figure 4b).

From the analysis results that the total required energy is not affected by the uncertain parameters for a number of dates. In fact, for a mission that starts on a day $\in[1,33] \cup[90,225]$ $\cup[276,365]$, nominal energy is equal to the maximum energy $(1540 \mathrm{Wh})$ and they depend only on the burn duration (LAE). However, from day 34 to day 89 and from day 226 to 275 the

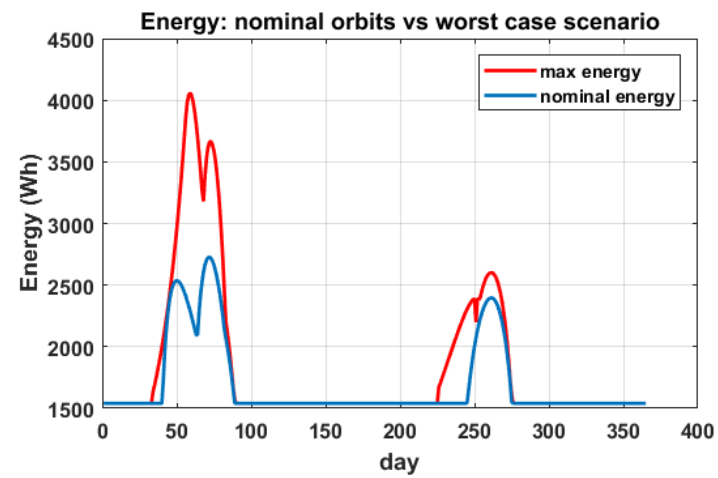

(a)

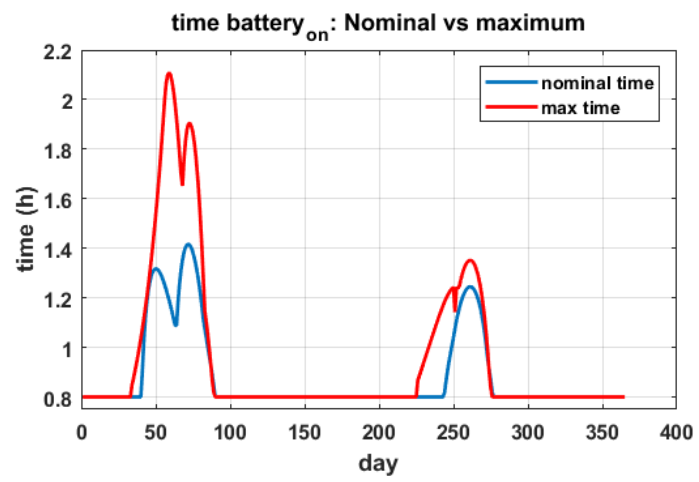

(b)

Fig. 4. First analysis: each day of 2019 has been considered for the satellite launch; for each day, the nominal and worst case scenario have been evaluated for the energy requirement(Figure 4a) and time of eclipse (Figure 4b).

energy requirement is strongly influenced by the uncertainties on the orbits and day 59 is certainly the most affected. From the minmax solution battery D results to be the best one for all the possible mission times. Figure 5 shows that the mass (nominal and maximum) of battery $\mathrm{D}$ is the lowest for all days of the year.

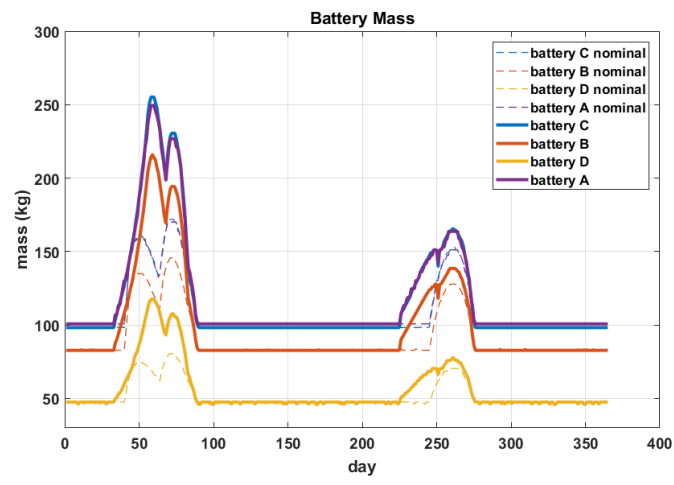

Fig. 5. Comparison of the nominal and maximum masses obtained with the four different batteries for each day of 2019.

2) H-Decomposition: The full Belief curve of the battery sizing problem requires $2^{31}=2.1475 \cdot 10^{9}$ maximisations (one for each FE), thus it is intractable. The computational cost of 
the decomposition approach, as explained in Section V-B, is:

$$
N_{F E}^{D E C}=N_{s} \sum_{i=1}^{m_{u}} N_{F E, i}^{u}+\sum_{i=1}^{m_{c}} N_{F E, i}^{c}=320+2 N_{s} .
$$

Figure $6 a$ shows the partial Belief curve of the mass $M$ considering all the FE $\theta_{k, 26} \in \Theta_{26}$ of the coupled variables $\mathbf{u}_{26}$ that influence both sub-systems two and six; the other partial curves are not significant for the final reconstruction because, as explained in Section V-B, the uncertainty on the nodes one, three, four and five (and the corresponding orbits) have no influence on the value of the objective function $M$. Finally Figure $6 \mathrm{~b}$ shows the reconstructed Belief curve obtained with the h-decomposition approach with five samples in the space of the coupled vectors $\mathbf{u}_{i 6}$. Thus, according to (30), 330 different optimisation have been run, strongly reducing the computational cost of the exact evaluation.

For a convergence analysis of the h-decomposition please refer to [3].

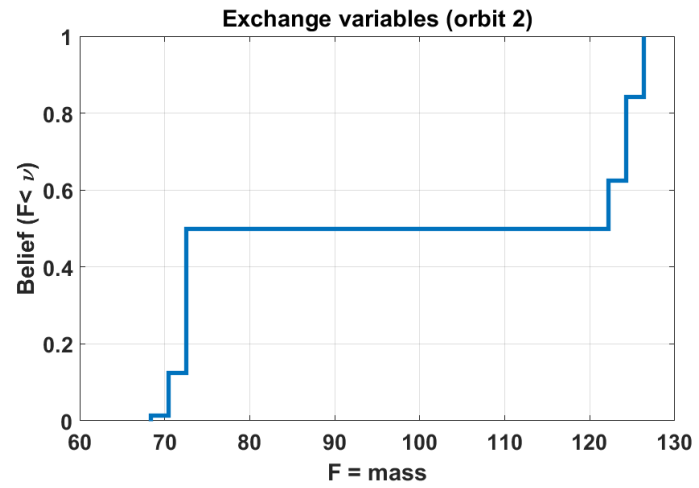

(a)

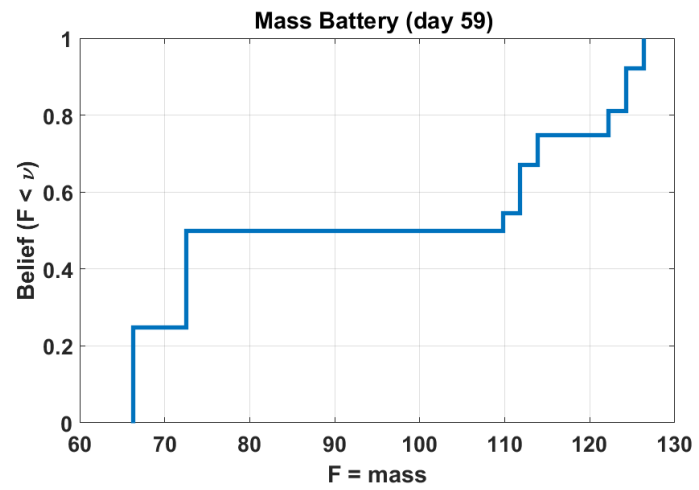

(b)

Fig. 6. Partial Belief curve, Figure 6a, of the coupled vector $\mathbf{u}_{26}$ and Belief curves, Figure $6 \mathrm{~b}$, of the spacecraft' mass for the day 59 obtained with 5 samples and then 330 optimisations.

\section{Validation}

A validation of the correctness of the results can be obtained in this way. Decompose the min-max problem in three steps:

- Fix a starting day for the mission: $\hat{t}$;

- Maximise the energy requirement over the orbit uncertainty:

$$
\max _{\mathbf{u} \in U} E(\hat{t}, \mathbf{a}, \mathbf{e}, \mathbf{i}, \mathbf{\Omega}, \boldsymbol{\omega}, \boldsymbol{\theta})
$$

- Minimise the mass over the design parameters (type of battery and voltage):

$$
\min _{\substack{\mathbf{d} \in D \\ t=\hat{t}}} M(\mathbf{d}, \mathbf{a}, \mathbf{e}, \mathbf{i}, \boldsymbol{\Omega}, \boldsymbol{\omega}, \boldsymbol{\theta}, \widehat{\eta})
$$

with $\widehat{\eta}=\min (\eta)$.

For $\hat{t}=59$ and evaluated $\operatorname{argmax}_{\mathbf{u} \in U} E$ (the red curve in Figure $4 \mathrm{a}$, the minimum Mass of the battery (Figure 5) corresponds to battery D and it is $126.3 \mathrm{~kg}$.

\section{CONCLUSION}

In this paper we proposed a new approach to optimise complex systems in space systems engineering, affected by epistemic uncertainty. We used Dempster-Shafer theory of Evidence to model uncertainty and Evidence Network Model (ENM) to model the system and its interconnections. From the battery sizing problem one can see that: the proposed constrained min-max scheme produces correct results and the decomposition approach delivers an approximation of the Belief nearly in polynomial time with a low exponent. Future work will include a statistical analysis of the robustness of the constrained minmax algorithm and an accuracy analysis of the approximated Belief curves.

\section{ACKNOWLEDGMENT}

This work is partially supported by the ESA NPI grant ref. TEC-ECN-SoW-20140806, H2020-MSCA-ITN2016 UTOPIAE, grant agreement 722734 and ESA/ITI project B00016563 Contract No. 4000116741/16NL/MH/GM. The authors gratefully acknowledge the support of Surrey Satellite Technology (SSTL), particularly Kathryn Graham and Kim Birkett, and ESA, particularly Harold Metselaar.

\section{REFERENCES}

[1] J. C. Helton Uncertainty and sensitivity analysis in the presence of stochastic and subjective uncertainty, Journal of Statistical Computation and Simulation, 1997, vol. 57, pp. 3-76

[2] W.L. Oberkampf and J.C. Helton, Investigation of Evidence Theory for Engineering Applications, AIAA 2002-1569, 4th Non-Deterministic Approaches Forum, 22-25 April 2002, Denver Colorado.

[3] M. Vasile, G. Filippi, C. Ortega and A. Riccardi, Fast Belief Estimation in Evidence Network Models, EUROGEN 2017.

[4] G. Shafer, A mathematical theory of evidence, Princeton University Press, 1976.

[5] M. Vasile On the Solution of Min-Max Problems in Robust Optimization, The EVOLVE 2014 International Conference, 2014

[6] M. Vasile, E. Minisci and M. Locatelli, An Inflationary Differential Evolution Algorithm for Space Trajectory Optimisation, IEEE Transactions on Evolutionary Computation, volume 15, number 2, April 2011.

[7] M. Di Carlo, M. Vasile and E. Minisci, Multi-population inflationary differential evolution algorithm with Adaptive Local Restart, IEEE Congress on Evolutionary Computation (CEC), 2015

[8] S. Alicino and M. Vasile, Evidence-based Preliminary Design of Spacecraft, $6^{\text {th }}$ International Conference on Systems \& Concurrent Engineering for Space Applications, October 2014.

[9] M. Vasile, On the Solution of Min-Max Problems in Robust Optimisation, The EVOLVE, 2014.

[10] M. R. Patel, Spacecraft Power Systems, CRC Press, 2005.

[11] D. A. Vallado, Fundamentals of Astrodynamics and Applications, Space Technology Library, 2007. 